\title{
Transmissão de energia elétrica em ultra alta tensão: o Complexo Hidrelétrico Belo Monte
}

\author{
Eletric power transmission in ultra high voltage: Belo Monte Dam Hydroeletric Complex \\ Rafael Garcia Conceição*, José Antonio Bento de Andrade, Carlos Henrique Ferreira, Carlos Jesivan \\ Alburqueque
}

Como citar esse artigo. Conceição RG, Andrade JAB, Ferreira $\mathrm{CH}$, Albuquerque CJ. Transmissão de energia elétrica em ultra alta tensão: o Complexo Hidrelétrico Belo Monte. Revista Eletrônica Teccen. 2016 Jan./Jun.; 09 (1): 66-74.

\section{Resumo}

A transmissão em ultra alta tensão é uma tecnologia que esta sendo desenvolvida na China e na Rússia e chega ao Brasil de forma mais marcante com a construção da UHE (Usina Hidrelétrica) Belo Monte permite a transmissão de grandes blocos de energia, para regiões mais afastadas dos centros de geração. O presente trabalho trata dos principais conceitos técnicos de transmissão em corrente continua assim como em transmissão em UAT (Ultra alta tensão) e tem como objetivo apresentar um estudo panorâmico sobre esta modalidade de transmissão de energia elétrica em corrente continua, bem como apresentar, como referencia principal para analise, o sistema de transmissão projetado para transportar energia da UHE Belo Monte. Para realizá-lo foi feita ampla pesquisa a cerca do projeto e execução do empreendimento UHE Belo Monte, assim como todos os aspectos técnicos e operacionais relativos à transmissão de energia, a partir dessa central de geração. O trabalho permite conhecer as principais vantagens da utilização da transmissão de grandes blocos de energia em corrente continua.

Palavras-Chave: Linhas de transmissão; Corrente contínua; Ultra Alta Tensão; Belo Monte.

\begin{abstract}
Transmission in ultra high voltage is a technology being developed in China and Russia and arrives in Brazil in a more marked form with the construction of the UHE (Hydroelectric Power Plant) Belo Monte and allows the transmission of large blocks of energy, for the more remote regions from the generation centers. The present work deals with the main concepts of technical transmission in continuous current as well as in transmission in UAT (Ultra High Voltage). This research has the objective to present a wide study on this modality in electricity transmission, as well as submit, as the main reference for review, the transmission system designed for energy transmission used at the UHE Belo Monte. Extensive research about the design and implementation of the venture UHE Belo Monte, as well as all the technical and operational aspects relating to energy transmission from this central generation was conducted. This paper enables the reader to recognise the main advantages of the use of the transmission of large blocks of energy in continuous current.

Keywords: Transmission lines; Continuous current; Ultra High Voltage; Belo Monte.
\end{abstract} absurdamente, com isso, cresce também a demanda de energia elétrica no país, principalmente em regiões mais afastadas dos grandes centros urbanos. Surge, pois, a necessidade do transporte da energia elétrica, através de distancias não muito pequenas. Contudo, alguns locais de grande população vão sendo desenvolvidos no trajeto, o que acaba acarretando maiores dificuldades no transporte de energia elétrica.

Segundo Carvalho (2012), o custo do transporte aumenta com a distância a ser vencida e diminui com a quantidade de energia a ser transportada. Qualquer estudo de viabilidade econômica de um aproveitamento hidroelétrico devera equacionar custo de produção e custo de transporte de energia produzida.

Uma das principais vantagens no uso de transmissão em ultra-alta tensão é de ordem econômica, ou seja, economizando em linhas ou cabos a fim de cobrir os aumentos dos custos das estações.

O crescimento de novas técnicas utilizadas para transportar energia elétrica torna cada vez mais compreensível, a utilização de transmissão em corrente continua aparece em um cenário onde a vantagem em relação a transmissão em CA (Corrente Alternada) é bastante elevada, considerando o uso aproveitado da melhor qualidade de energia transportada. O surgimento de novas tecnologias para maximizar as vantagens e diminuir os custos em relação as estações conversoras, vem se tornado cada vez mais eficaz.

Este trabalho tem por finalidade apresentar um estudo panorâmico sobre conceitos gerais de transmissão de energia elétrica em corrente contínua em ultra-alta tensão, bem como apresentar como referência principal

Afiliação dos autores: Universidade Severino Sombra, Vassouras-RJ, Brasil.

* Endereço para correspondência: Universidade Severino Sombra, Av. Exped. Oswaldo de Almeida Ramos, 280 - Centro - Vassouras, RJ - CEP $27700-000$. E-mail: eng.rafaelgarcia@live.com 
para análise o sistema de transmissão projetado para transportar energia da UHE Belo Monte até o sudeste do Brasil.

O trabalho foi realizado tomando por base o estudo do sistema HVDC (high Voltage Direct Current, Corrente Contínua em alta tensão) como foco na transmissão de grandes blocos e de todos os seus componentes. Primeiramente foi estudado todo o sistema em ultra alta tensão já existente bem como seus componentes e suas particularidades. Em seguida foram pesquisados os principais equipamentos para esse tipo de transmissão e suas aplicações, dando foco na construção de belo monte, demonstrando seus aspectos constitucionais, funcionais e operacionais.

\section{Corrente elétrica}

Segundo Gussow (1997), o nome corrente é dado a todo movimento ou fluxo de elétrons. Para se produzir os elétrons devem se deslocar pelo efeito de uma diferença de potencial. A corrente é representada pela letra I. A unidade fundamental com que se mede a corrente é o ampere (A). Um ampere de corrente é definido como o deslocamento de um Coulomb através de um ponto qualquer de um condutor durante um intervalo de tempo de um segundo.

\section{Corrente contínua}

Corrente continua é a corrente unidirecional de mesma polaridade de tensão, que passa através de um condutor ou de um circuito em um único sentido, essa corrente é encontrada nas saídas de pilhas e baterias.

A tensão fornecida por essas fontes é chamada de tensão de corrente continua ou de tensão DC (Direct Currente, Corrente Contínua). Uma fonte de tensão contínua pode variar o valor da sua tensão de saída, mas se a polaridade for mantida, a corrente fluirá somente num sentido (GUSSOW, 1997).

\section{Corrente alternada}

O termo alternada se dá pelo fato de ocorrer a alternância de tensão e corrente durante um determinado valor de tempo.

Boylestad (1997) classifica Corrente alternada indica apenas que o valor da tensão ou da corrente alterna (oscila) regularmente entre dois níveis. Na distribuição de energia elétrica para uso domiciliar e industrial só é utilizada a tensão alternada senoidal.

$\mathrm{Na}$ Figura 1 é possível representar a forma de onda alternada onde o eixo zero é uma linha horizontal que passa pelo centro. As variações verticais na onda de tensão mostram as variações do modulo. As tensões acima do eixo horizontal têm polaridade positiva, enquanto as tensões abaixo do eixo horizontal tem polaridade negativa.

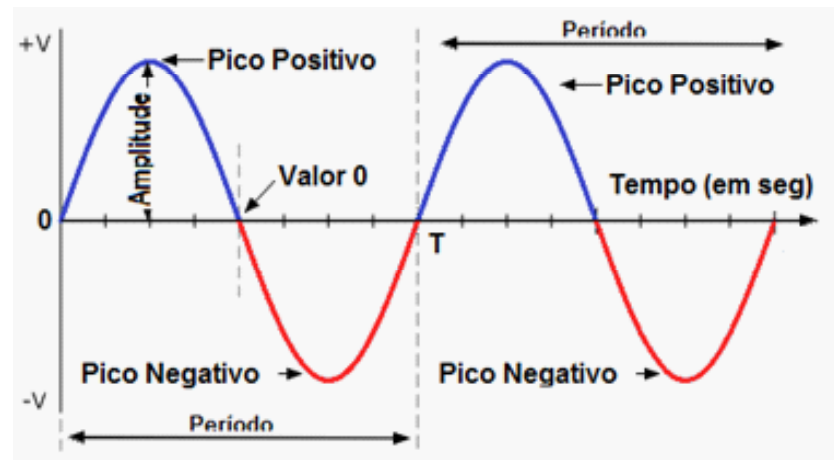

Figura 1. Forma de Onda Alternada. Fonte: BOYLESTAD, 1997.

\section{Tiristor}

Tiristor corresponde a um nome genérico de uma família de dispositivos semicondutores que possuem, basicamente, quatrocamadas(PNPN), comcaracterísticas biestáveis em seu funcionamento, isto é, permanecem indefinidamente conduzindo ou bloqueados, a menos que fatores externos os levarem a uma mudança de estado. Estes componentes têm aplicações dentro da Eletrônica Industrial ou Eletrônica de potência, gerando pulsos de controle para outros tiristores, convertendo de CA para CC (Corrente Contínua), CC para CA ou CC para $\mathrm{CC}$, chaveando grandes, principalmente motores, eletroímãs, aquecedores e hoje está presente também, nos controles de temperatura das duchas ou chuveiros. Dentro desta família de dispositivos, o principal e mais importante, é o SCR (Silicon Controlled Rectifier ou Retificador Controlado de Silício).

Ahmed (2000) Caracteriza um tiristor como sendo dispositivos semicondutores de potência com quatro camadas PNPN, usados como chaves eletrônicas. A principal vantagem que oferecem é converter e controlar grandes quantidades de potência em sistemas AC (alternative Current, Corrente Alternada) ou DC, utilizando apenas uma pequena potência para controle.

\section{Retificador controlado de silício}

Segundo Ahmed (2000), O retificador controlado de silicio (SCR) é o controlador elétrico de potência com uso mais difundido, isso se deve a sua ação de chaveamento rápido, ao seu pequeno porte e aos seus altos valores nominais de corrente e de tensão.

Na Figura 2, mostra a estrutura de um SCR, formado por 4 camadas PNPN e uma porta gate, que tem como função controlar o ângulo de disparo.

Na Figura 3 está o simbolo elétrico de um SCR, onde é possivel ver que um SCR possui três terminais:o 
anodo e o Catodo são os de potência, enquanto a porta (G) é o de controle.

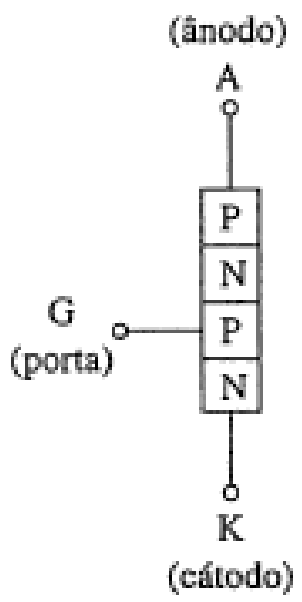

Figura 2. Estrutura de um SCR. Fonte: AHMED, 2000.

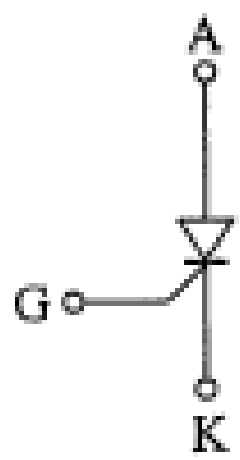

Figura 3. Símbolo elétrico SCR.

Fonte: AHMED, 2000.

Quando o SCR está diretamente polarizado, isto é, quando o anodo é positivo em relação ao catodo, uma tensão positiva na porta, com relação ao catodo, passa o SCR para o estado ligado. Entretanto, não é a porta que desliga a corrente no SCR. Ela é desligada quando se interrompe a corrente do anodo. De modo similar ao que ocorre com um diodo, o SCR bloqueia a corrente na direção inversa (AHMED, 2000).

\section{Transmissão de energia elétrica}

Para garantir maior eficácia na produção de energia, as centrais de geração usualmente são construídas distantes dos consumidores finais de energia, por esse motivo, a energia produzida precisa ser transportada por longas distancias por um sistema de transmissão.

Este sistema é composto basicamente, por cabos aéreos revestidos por um material isolante, ligados à grandes torres metálicas por estruturas isolantes de vidro ou porcelana. Todo este conjunto é chamado de rede de transmissão (Baroni, 2012).

\section{Transmissão de energia elétrica em corrente alternada}

Em um sistema em corrente alternada, ocorre uma conversão relativamente simples, apenas para subtransmitir para os devidos consumidores, que possui um transformador em corrente alternada que exige altos níveis de isolamento e grandes níveis de potência. Por ser um sistema simples, esse sistema requer pouca manutenção.

A rede de transmissão transporta uma grande quantidade de energia elétrica por longas distâncias, mas antes de chegar aos centros de consumo, esta passa por uma subestação abaixadora, que tem a função de abaixar o nível de tensão, para que esta esteja em um nível adequado para o abastecimento de pequenas cidades ou consumidores de grande porte (Baroni, 2012).

\section{Transmissão de energia elétrica em corrente contínua}

O sistema de transmissão em corrente continua é composto por uma estação conversora, que converte a tensão em corrente alternada dos centros geradores para corrente contínua, e uma estação inversora, que tem como função reverter essa tensão em corrente contínua novamente para corrente alternada, para o processo de subtramissão aos consumidores (Kimbark, 1971).

Nos sistemas de transmissão em corrente continua onde denominamos HVDC, a corrente e tensão são retificadas e depois invertidas novamente para alternada, desta forma as linhas transportam energia usando corrente continua.

A transmissão em HVDC tem como principal característica o fator de que a tensão se mantém constante e da não alteração de polaridade durante todo o processo. Alem disso, através de rápida modulação no processo de conversão, a transmissão DC pode ser usada para amortecer oscilações da rede AC e então melhorar a estabilidade do sistema (Kimbark, 1971).

A Figura 4 ilustra a diferença entre transmissão HVDC e em ATCA (corrente alternada em alta tensão), onde basicamente se diferem no controle de corrente, que em HVDC é necessário somente um par de cabos, válvulas retificadoras e reatores, enquanto que para a transmissão em ATCA são necessários no mínimo 3 cabos e reatores "shunt" para evitar as sobretensões durante o chaveamento da linha em função da alta capacitância (Silva, 2011). 


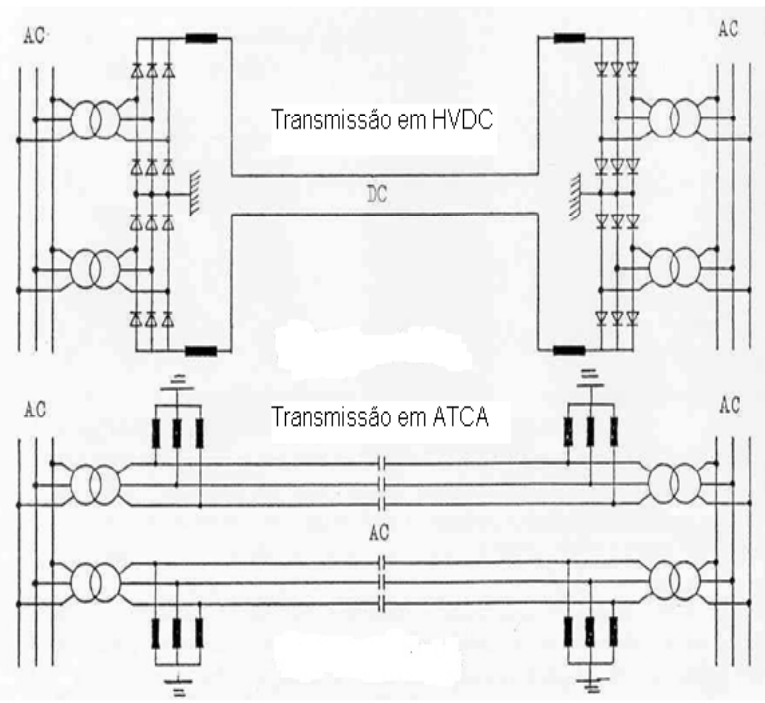

Figura 4. Opções típicas de transmissão em HVDC e ATCA Fonte: SILVA, 2011

\section{Sistema de conversão}

Segundo Sato (2013), existem dois tipos de conversão utilizados nessa tecnologia de transmissão em corrente contínua: LCC (Line Commutated Converter) foi a primeira desenvolvida e é a mais utilizada, esta utiliza tiristores como principal elemento de comutação, atingindo altos níveis de capacidade de transmissão de potência, altas tensões e altas correntes. Outro tipo de conversor é o VSC (Voltage Source Converter) e foi desenvolvido mais recentemente, onde seu principal elemento é um IGBT (Insulated Gate Bipolar Transistor, Transistor Bipolar de Porta Isolada), logo é possível atingir alto nível de controle na conversão de potência e grandes velocidades.

$\mathrm{Na}$ transmissão em ultra alta tensão são utilizados conversores do tipo LCC, pois é constituído por uma ponte retificadora de 12 pulsos, onde os conversores dependem exclusivamente da rede no qual são conectados para ocorrer a comutação.

Carvalho (2012) relata que o processo de conversão é realizado por uma comutação controlada da condução de corrente entre os distintos dispositivos semicondutores, a tensão no terminal da ponte conversora é composta por trechos de tensões fase-fase do lado CA.

Basicamente, ilustrado na Figura 5, um conversor trifásico tem como função principal, o papel de chave ou válvula, liberando ou restringindo a passagem de corrente elétrica.

Na Figura 5, as Chaves 1 e 2 conduzem corrente L1 e L2, iguais à id. Com isso a tensão Vf se torna igual a tensão da fase e enquanto a tensão 1 é igual a tensão da fase c, resultando em uma tensão $\mathrm{Vd}$ igual a Ua - Uc. A próxima chave a conduzir é a de numero 3 , cuja tensão de polarização corresponde a Ub - Ua. Quando esta se torna positiva a chave 3 poderá iniciar o processo de tomada de corrente, fazendo com que a chave 1 se apague, e que a tensão Up se torne igual a $\mathrm{Ub}$, e consequentemente Ud igual a Ub - Uc (Carvalho, 2012).

Esses conversores podem ser formados por seis pulsos ou doze pulsos como mostrado abaixo. A Figura 6 mostra tiristores organizados em pontes conversoras de 6 pulsos e cada uma dessas pontes é constituída por tirisitores dispostos em série.

A Figura 7 mostra a organização de tiristores ligados em série formando uma ponte de 6 pulsos. Essas pontes de 6 pulsos são ligadas em série organizando assim pontes de 12 pulsos.

Um sistema de 12 pulsos é usado para reduzir mais ainda a tensão de ondulação na saída DC e aumentar a frequência de ondulação (Ahmed, 2000).

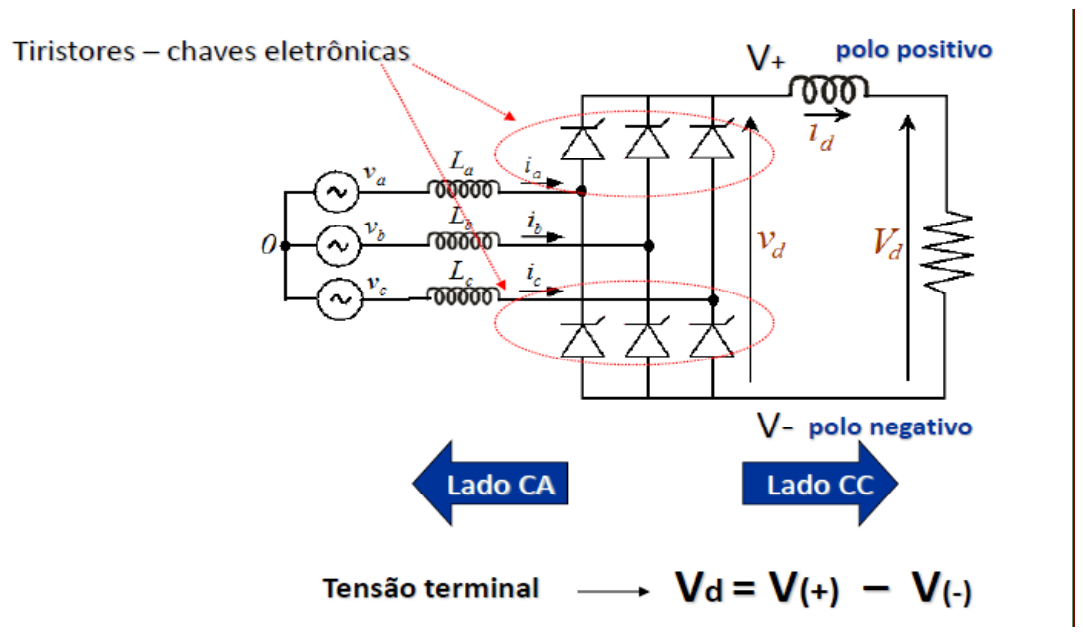

Figura 5. Conversor Trifásico em ponte.

Fonte: Carvalho, 2012. 


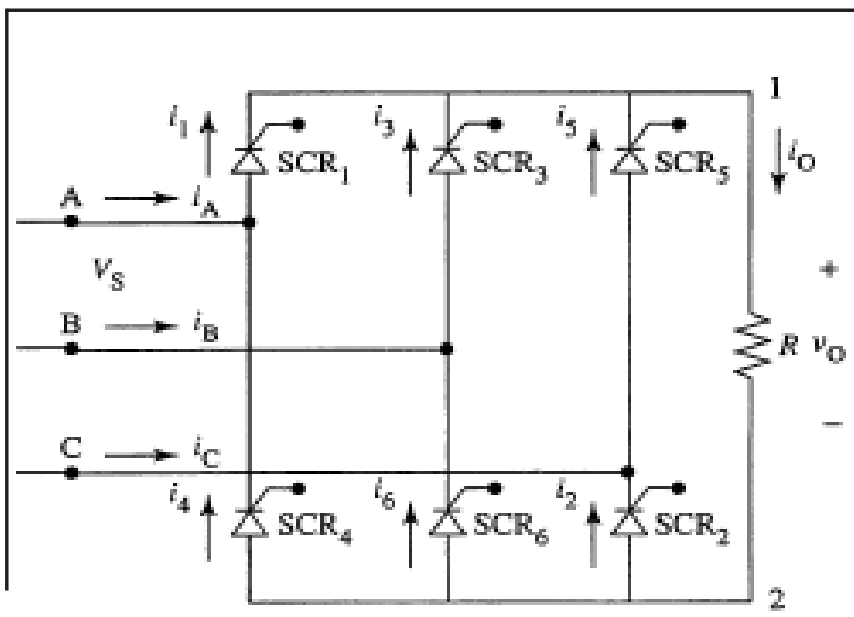

Figura 6. Ponte Retificadora de 6 pulsos.

Fonte: Ahmed, 2000.

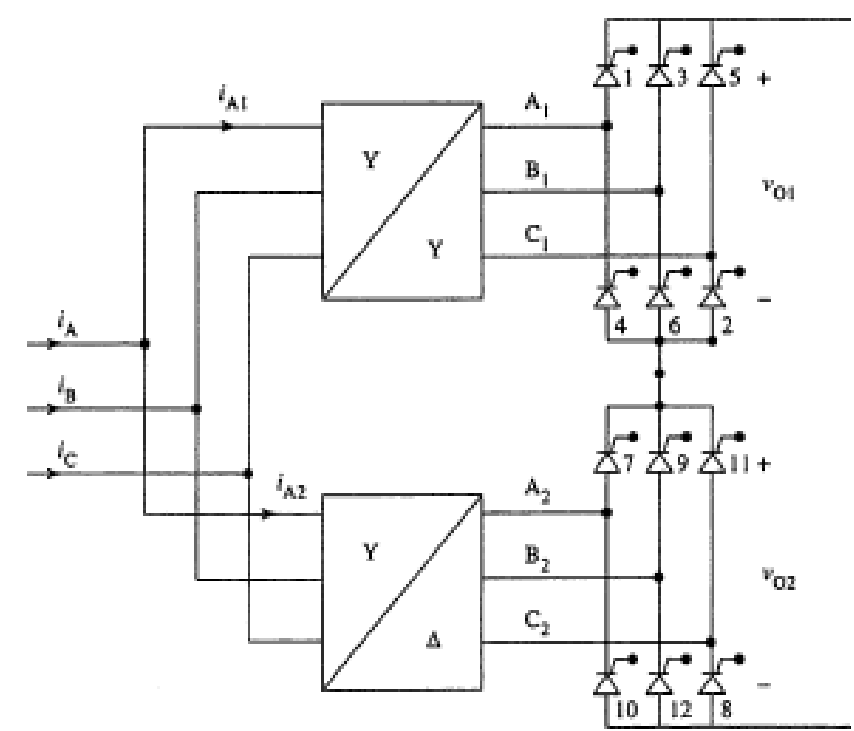

Figura 7. Ponte Retificadora de 12 pulsos

Fonte: Ahmed, 2000.

\section{Sistema de inversão}

Ao completar todo o processo de transmissão em grande escala, o sistema deve ser convertido novamente para corrente alternada, para a subtransmissão dos consumidores.

Kimbark (1971), descreve essa saída dizendo que o nível DC será convertido novamente em AC por um conversor em ponte funcionando agora como inversor. Neste caso o ângulo de retardo é de $180^{\circ}$.

A relação entre os ângulos $\alpha, \mu, \square$ É dada por: $=180^{\circ}-\alpha-\mu$

Logo concluímos que a estação que funciona como retificadora procura ter um ângulo de retardo próximo de $0^{\circ}$, a estação que funciona como inversora procura ter um ângulo próximo de $180^{\circ}$, com a maximização da diferença de tensão, com isso uma potência maior é transferida.

\section{Sistema de proteção}

Para proteção de todo o sistema, o projeto de níveis de proteção de pára raios, leva a um nível de isolamento para todos os elementos da estação conversora, pois há a possibilidade de surtos de tensão que podem ser ocasionados pela incidência de descargas atmosférica de ambos os lados, tanto em CC ou em CA, além de surtos por chaveamento são gerados no lado CA pela manobra de disjuntores, com ou sem faltas, ou até mesmo sobre tensões ferrorressonantes podem resultar de saturação de transformadores seguida de faltas (Carvalho, 2012).

\section{Tipos de ligações em corrente contínua}

A Transmissão em corrente contínua pode ser escolhida, diferenciando - se uma da outra a partir dos números de condutores e de sua polaridade.

A Figura 8 apresenta o exemplo de uma ligação unipolar, possuindo um único condutor com retorno pelo terra.

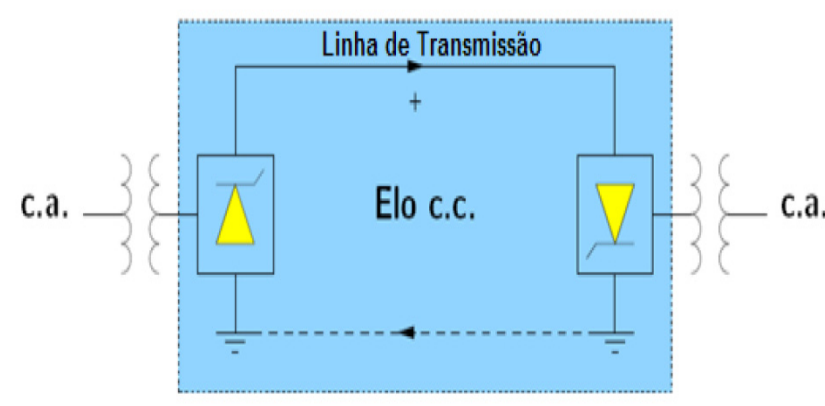

Figura 8. Sistema Unipolar Elo CC. Fonte: Reis, 2013.

Já a figura 9 mostra um exemplo bipolar onde há dois condutores um positivo e um negativo. Cada terminal tem dois conversores de tensão nominal igual em serie no lado DC. Então pontos (junções entre conversores) estão fundamentados em uma ou ambas as extremidades. Neutros são aterrados, e os dois polos podem operar de forma independente. Normalmente eles operam em igual; então não há corrente de terra. Nesse exemplo em caso de uma falha em um condutor, o outro condutor com retorno de terra pode transportar até metade da carga nominal.

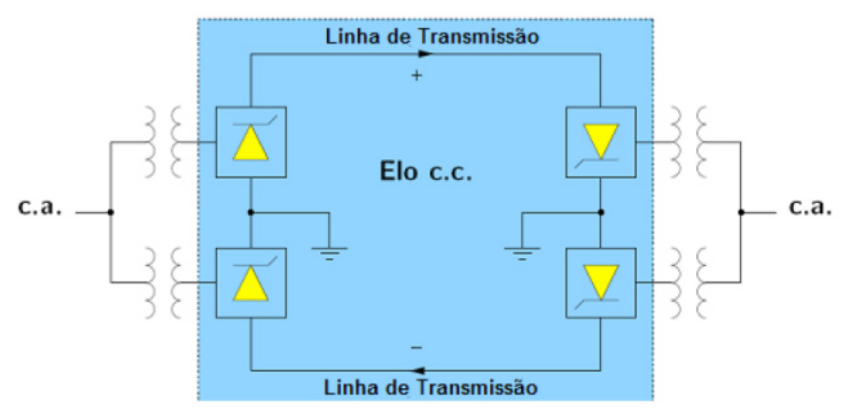

Figura 9. Sistema Bipolar, Elo CC.

Fonte: Reis, 2013. 
Além disso, Kimbark (1971), relata que existe o tipo homopolar que em sua estrutura possui dois ou mais condutores todos tendo a mesma polaridade, geralmente negativo, e sempre opera com retorno de terra. No caso de uma falha de um condutor, o conversor de outra está disponível para a ligação ao condutor restante.

\section{Transmissão de energia elétrica em corrente contínua em ultra alta tensão}

Uma Transmissão em ultra-alta tensão segue várias práticas estabelecidas para projetos HVDC existentes, no entanto com a potência e voltagem mais elevadas. Sua formação possui um arranjo com uma única configuração de ponte de 12 pulsos por polo, como mostrado na Figura 7.

Consiste em uma ligação em série de duas pontes de 12 pulsos com 400kv de tensão nominal cada uma. A fim de permitir a transferência de energia interrupta durante a conexão e desconexão de certos indivíduos, interruptores de desvio, chaves seccionadoras e chaves by-pass (Haeusler; Huang; Papp, 2008).

A grande vantagem em usar esse arranjo, se dá no aumento da disponibilidade de energia, logo uma vez que qualquer falha de um único grupo afeta apenas $25 \%$ da capacidade de potência instalada.

Um dos fatores importantes no dimensionamento de uma linha de transmissão em UAT se deve a sua característica física, o que acaba resultando no custo total e final de transporte de energia. Uma Transmissão em UAT exige em sua estrutura, materiais específicos e poucos convencionais, diferenciando da transmissão convencional em corrente continua.

O custo de transmissão está diretamente relacionado com a distância a ser transportada, pois logo, quanto maior a distância, menor é o custo das estações em HVDC, levando em consideração, que, de acordo com que se aumenta a distância, ganhamos em potência, diminuindo o custo com isolamento e principalmente com o custo de condutor.

Outro ponto importante no dimensionamento, é o fator diferencial dos tiristores, que são considerados sua corrente de curto circuito, que é dada pela relação da reatância do transformador com a corrente $\mathrm{CC}$ nominal do sistema.

\section{Característica física}

A aplicação UHVDC (ultra high voltage direct current, Corrente Contínua em Ultra-alta tensão) conta com arranjo modular com tiristores empilhados e dissipadores de calor. As torres são válvulas dispostas como válvulas duplas, suspensas no teto do salão de válvula. Assim, seis torres formam um grupo de 12 pulsos. Como mostra a Figura 10 (Haeusler; Huang; Papp, 2008).

Haeusler (2008) descreve que cada uma das duas válvulas em uma estrutura de dupla válvula consiste de duas unidades modulares de válvula. Assim, uma torre tem quatro unidades modulares, cada unidade modular da válvula por sua vez, inclui duas seções de válvulas ligadas em série e cada seção de válvula compreende 15 níveis de tiristores. Portanto, uma válvula de tiristor com duas unidades modulares compreende quatro seções de válvulas e é composto por 60 serie de tiristores ligados. Uma seção de válvula também inclui os dissipadores de calor do tiristor.

A Figura 10 mostra a imagem de uma unidade múltipla de válvulas, que possui duas seções de válvulas ligadas em série que compreendem exatamente quinze níveis de tiristores.

\section{Reatores de alisamento}

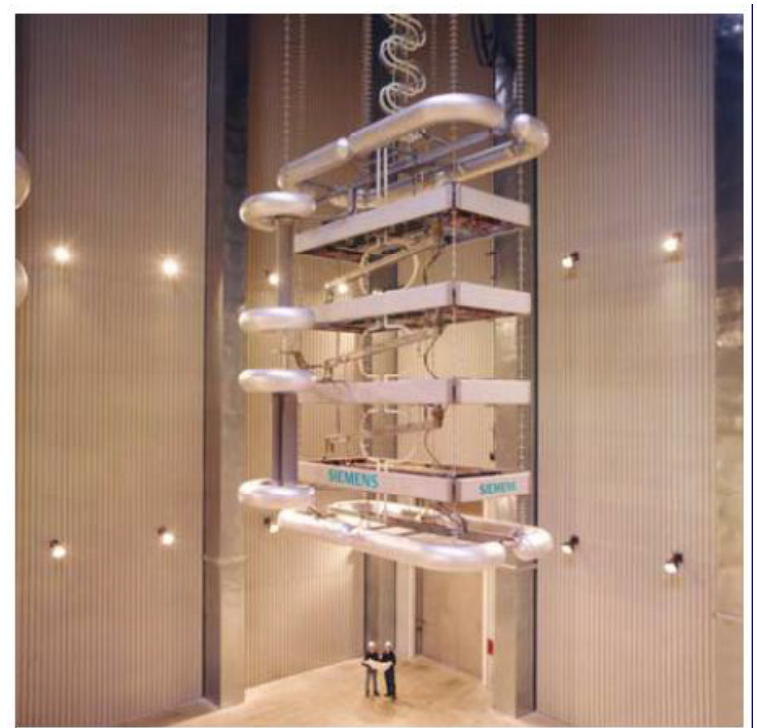

Figura 10. Unidade Múltipla de Válvula.

Fonte: HAEUSLER, 2008.

Uma diferença importante com a transmissão convencional é a disposição dos reatores de alisamento. A indutância total de $300 \mathrm{mh}$ é dividida em duas partes e instalada em locais diferentes. Esses reatores devem ser do tipo seco fazendo com que a indutância obtida seja feita ligando varias bobinas de reatores em série. Por questões econômicas, $50 \%$ seve ser instalado no barramento neutro e os outros $50 \%$ em barramento cc de alta tensão. Além de aspectos econômicos para os reatores de alisamento próprio, outro fator que é benéfico para o dimensionamento do sistema é decorrente na queda de tensão através de harmônicos, pois o barramento neutro do reator (ligado a linha do eletrodo) reduz a tensão harmônica a terra, isso afeta especialmente o estado estacionário de ondulação da tensão para terra dos transformadores conversores de $800 \mathrm{kv}$ (Haeusler, 2008).

A escolha do tipo seco para tecnologia UHVDC se 
deve quanto a razões econômicas e quanto a técnicas. O argumento técnico mais convincente é o da simplicidade de isolamento do reator para terra. Em HVDC, reatores de isolamento são ligados em série com terminais do conversor. Ao ser ligado ao terminal do polo, o reator está em plena potência em relação a terra.

\section{Estações conversoras}

As estações conversoras são projetadas para transmitir grande concentração de energia sem sistema de refrigeração redundante em serviço de 1,1pu de potência nominal com redundante sistema de arrefecimento em serviço com temperaturas ambiente máximas. Além disso, a transmissão UAC permite uma sobrecarga de 2 horas de $1,1 \mathrm{pu}$ de potência nominal, e ate $1,2 \mathrm{pu}$, sem e com respectivamente, equipamentos de refrigeração redundante em funcionamento (Zhang; Haeusler; Rao; Shang; Shang, 2007).

A capacidade de sobrecarga de tempo curto é importante para algumas contingências, como a energia modulação após falhas do sistema de corrente alternada.

A fim de aperfeiçoar o filtro e o conversor transformador, deve-se projetar a capacidade das válvulas a operar em ângulos de disparos elevados, combinados com uma ampla gama de comutação. Além disso, a cooperação com grupos de válvulas individuais fora de serviço é possível, ou seja, a operação a $400 \mathrm{kv}$ $(50 \%)$ (Haeusler, 2008).

\section{Coordenação de isolamento}

Nunes (2006), afirma que a coordenação de isolamento de uma linha de transmissão consiste na seleção de distâncias de isolamento adequadas às sobretensões esperadas para o sistema, levando-se em consideração a característica de possíveis equipamentos protetores. No entanto, seria muito dispendioso economicamente construir uma linha que suportasse todas as sobretensões possíveis. Deste modo, a escolha dos isolamentos deve ser realizada de forma que se minimizem os custos e se obtenha uma determinada probabilidade de desligamento da linha para cada evento transitório, expressa como um risco de falha aceitável.

Em relação ao isolamento externo, outro aspecto importante é a seleção de dispersão adequada, bem como de material de isolamento adequado.

A partir de 500kv, regimes HVDC são conhecidas por isoladores de porcelana, instalados em áreas poluídas, pois exigem altas distâncias de escoamento específicas, por exemplo, até $60 \mathrm{~mm} / \mathrm{kV}$ para buchas do tipo de porcelana de parede montadas horizontalmente.

De acordo com Haeusler; Huang; Papp (2008), alguns materiais foram desenvolvidos para ajudar na redução de problemas causadas pela poluição flashovers. Materiais com as superfícies de silicone hidrofóbico por exemplo, impedem a construção de camadas condutoras nas superfícies de isoladores poluídos. Em comparação com os requisitos de porcelana, esse material reduz até $75 \%$ da distância de dispersão, este aspecto é muito importante, pois ajuda a evitar dimensões excessivas de equipamento principal.

\section{Estudo de Caso: o complexo hidrelétrico Belo Monte}

A usina hidrelétrica de Belo Monte situada no estado do Pará, é um projeto de planejamento energético brasileiro, que tem como objetivo acrescentar a potência de 11.233MW ao sistema interligado brasileiro (SIN), sendo $11.000 \mathrm{MW}$ na casa de força principal e $233 \mathrm{MW}$ na casa de força secundária.

De acordo com o Balanço Energético Nacional de 2011, o projeto Belo Monte adotará um sistema de canal inovador, que reduz o tamanho da área necessária para a geração de energia. $O$ barramento e o vertedouro principal ficarão no sítio Pimentel, no leito do rio Xingu, a cerca de $40 \mathrm{~km}$ da cidade de Altamira. A casa de força complementar também será instalada no sítio Pimentel. Por meio de canalização, parte da água do rio será desviada para a casa de força principal em Belo Monte, formando um reservatório de canais.

A UHE Belo Monte será a terceira maior hidrelétrica do mundo, ficando atrás das Três Gargantas (China) e de Itaipu Binacional; com grande capacidade e potencial para gerar muita energia, parte do que for gerado nos meses chuvosos será enviada para os estados das regiões Sudeste e Nordeste, principais consumidores de energia do país (Machado; Pereira; Ramos, 2011).

A UHE Belo Monte será integrada ao SIN (Sistema Interligado Nacional) através de sistemas de transmissão de uso exclusivo que interligarão as casas de força principal e complementar ao sistema interligado nacional.

\section{Características Belo Monte}

O sistema da UHE Belo Monte conta com três subsistemas específicos que são definidos pelo nível de tensão 500,230 e $69 \mathrm{kv}$.

O sistema que transportará em ultra alta tensão, é o de $500 \mathrm{kv}$, que transmitirá energia elétrica ate o sudeste do Brasil.

Possui duas estações conversoras (EC), uma em Xingu e outra em Estreito, para a conversão de corrente contínua em corrente alternada e vice-versa; dois eletrodos de terra; duas linhas de eletrodo (em tensão neutra), que ligarão os eletrodos ás ECs; sete estações repetidoras, assim, as estações conversoras farão o 
processo de converter $500 \mathrm{kV}$ em corrente alternada para $800 \mathrm{Kv}$ em corrente continua, para o estado do Rio de Janeiro e Minas Gerais, sendo novamente convertido para $500 \mathrm{kv}$ em corrente alternada para a interação com o sistema interligado nacional.

O projeto consiste em dois bipolos de transmissão aérea, com extensão total de 2086,9km SE (Subestação) Xingu/Estreito e $2439 \mathrm{~km}$ SE Xingu/T.Rio em corrente contínua, tensão $\pm 800 \mathrm{KV}$ e potência nominal de 4.000MW, ambos ligando em SE 500KV de Estreito (MG) e SE 500KV T.Rio, respectivamente.

Segundo o relatório de impacto ambiental de 2015, entre Xingu e Estreito serão usadas dois tipos de torre de transmissão, estaiadas, cerca de $80 \%$, e autoportante, cerca de $20 \%$, estima-se o uso de 3749 torres, estando dispostas a uma distância média de $560 \mathrm{~m}$ entre elas, trecho Anapu e Uruaçu, e de $550 \mathrm{~m}$, trecho Uruaçu e Ibiraci.

Isso se deve ao fato de por se tratar de transmissão de grandes blocos de potência, ganha-se em condutores, o que resulta automaticamente em ganhos físicos e ambientais.

A diferença entre as torres estaiadas e autoportantes se dá na vantagem econômica que as torres estaiadas garantem sobre as autorportantes, pois apresentam apenas uma fundação. Já as autoportantes podem ser montadas em áreas restritas, mas resultam em maior custo por necessitarem de quatro fundações.

$\mathrm{Na}$ Figura 11, a diferença entre as duas torres de transmissão, usando como exemplo uma transmissão de $400 \mathrm{kV}$

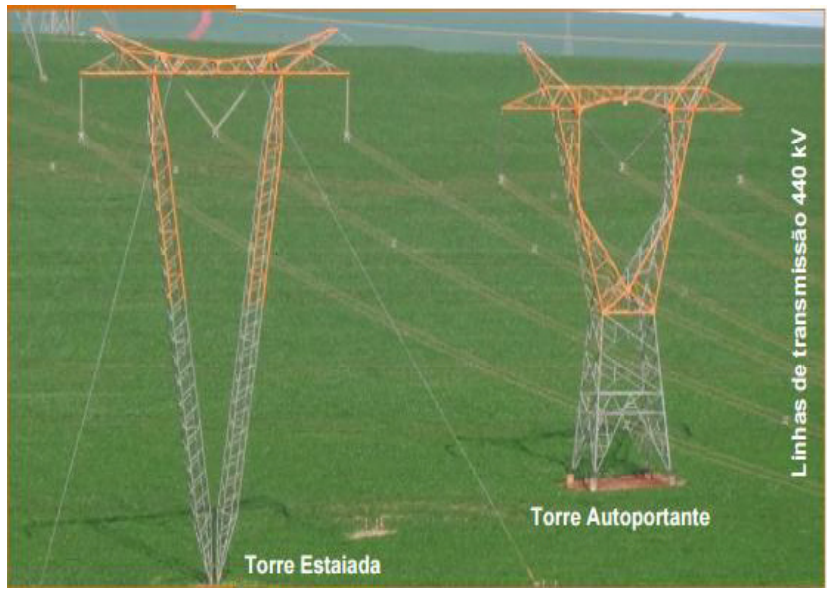

Figura 11. Torre Estaiada e Torre Autoportante $\pm 400 \mathrm{KV}$. Fonte: Rima, 2015.

\section{Análises e Discussão}

\section{Contribuição de Belo Monte para o SIN}

A UHE Belo Monte vai integrar o Sistema Interligado Nacional e, com isso, sua energia produzida vai contribuir para expansão da oferta em todo o País. Assim, na cheia do Rio Xingu, será possível produzir grandes quantidades de energia, promovendo a acumulação de água nos reservatórios das usinas de outras regiões, tirando proveito da sazonalidade hidrológica decorrente das dimensões continentais do País. Complementarmente, nos períodos de seca do rio Xingu, essas usinas com água armazenada suprirão a diferença da menor geração em Belo Monte (Machado; Pereira; Ramos, 2011).

Belo Monte terá como média a geração de 4.428MW, devido a variação da vazão do rio Xingu durante o ano. Porém continua sendo muito importante para o país, pois nenhuma hidrelétrica opera em potência máxima durante todo o ano. Outro ponto bastante importante para o ONS é o fato de enquanto houver vazão para ativar as turbinas em Belo Monte, outras usinas podem parar seu funcionamento, com o objetivo de economizar água nos reservatórios.

De acordo com a Agência Nacional de Energia Elétrica o SIN permite o intercâmbio de energia produzida por todas as usinas que compõem o sistema. No caso da UHE Belo Monte, a interligação ao SIN deverá ser feita por cinco linhas de transmissão em $500 \mathrm{kv}$, com extensão de aproximadamente $17 \mathrm{~km}$, operando em corrente alternada. O ponto de interligação com a Rede Básica será a subestação secionadora Xingu, que faz parte da interligação Tucuruí-Macapá-Manaus. A partir desta subestação partirão as interligações previstas com as regiões Sudeste/Centro-Oeste e Nordeste.

O Balanço Energético Nacional de 2015 apresenta que Belo Monte trará consigo várias vantagens durante sua construção: Produção de mais de $11.000 \mathrm{MW}$ de Energia Elétrica o que é essencial do ponto de vista de Engenharia Elétrica para evitar futuros apagões e garantir o crescimento da Economia. A energia gerada é suficiente para abastecer as casas de 26 milhões de pessoas, uma população equivalente a área Metropolitana de São Paulo. A Energia Hidrelétrica é abundante, barata e limpa, uma excelente opção se comparada às usinas nucleares, que são caras e perigosas, ou então as usinas a carvão, petróleo e gás, que são poluentes. Novas Linhas de Transmissão jamais usadas nos país, com o uso de tecnologia chinesa, vão tornar o Sistema Energético Brasileiro mais eficiente. O lago da Hidrelétrica de Belo Monte pode servir para criação de Fazendas de Peixes

A usina vai operar a fio d'água. Isso significa que a geração vai variar de acordo com a quantidade de água do Rio Xingu a cada período do ano. Ou seja, a usina vai gerar mais energia nas épocas de cheia e menos nos momentos de seca. Assim, a garantia física do empreendimento será definida pela EPE. A Casa de Força Principal, que ficará no Sítio Belo Monte, com capacidade mínima instalada de 11 mil MW e garantia assegurada de 4,418 mil MWmed. A casa de força complementar, no sítio Pimentel, terá capacidade de 233,1 MW e garantia assegurada de 151,1 MWmed. Do ponto de vista da relação entre capacidade instalada e 
área alagada (MW/km2), a UHE de Belo Monte será a terceiro do país, atrás apenas de Xingó e Paulo Afonso IV (ANEEL, 2015).

\section{O futuro da produção de energia do Brasil}

Segundo o Balanço Energético Nacional 2011, o consumo de eletricidade cresceu $47 \%$ na ultima década, se aproximando a 455,7 TWH em 2010. Estima-se, pelo plano decenal de expansão de energia, que para 2020 o consumo aumentará até $55,6 \%$, podendo chegar a 659 TWh. Para atender a toda essa demanda, foi realizado o planejamento e a contratação de um aumento de $51 \%$ da capacidade instalada, passando dos 113,327 MW em operação para 171,138 MW em 2020. Estas novas centrais serão, em sua maioria, grandes centrais hidrelétricas, $32.184 \mathrm{MW}$, mas também estão previstas centrais termelétricas movidas a combustíveis fósseis, centrais termonucleares (1.405 MW) e centrais movidas a fontes alternativas (18.009 MW), que incluem energia eólica, biomassa e pequenas centrais hidrelétricas.

\section{Conclusões}

Em todo o mundo, a demanda de energia elétrica cresce a escala e velocidade nunca imaginada por gerações passadas. Com a geração de energia tornandose cada vez mais distribuídas e um número crescente de instalações de geração localizadas longe dos centros de carga, é bastante importante o estudo de novas tecnologias para transmissão de grandes blocos de energia.

No presente trabalho apresentou-se fundamentos científicos caracterizando transmissão de energia elétrica, focando principalmente em transmissão em corrente contínua, enfatizando uma tecnologia jamais usada no Brasil, que permite a transmissão de grandes blocos de energia para grandes centros distantes, tomando como exemplo a transmissão em $\pm 800 \mathrm{KV} \mathrm{CC}$ da UHE Belo Monte, é possível ver, a importância que esse empreendimento trará para o Brasil, no que diz respeito a energia elétrica e sua importância ao SIN.

Foi possível concluir que esse sistema possui grandes vantagens em relação a transmissão em corrente alternada. Levando em consideração aspectos econômicos e ambientais.

\section{Referências}

Ahmed, A. Eletrônica de Potencia. São Paulo: Prentice Hall do Brasil LTDA, 2000

Balbierer, S.; Haeusler, M.; Ramaswami, V.; Hong, R; Chun, Sh; Tao, Sh; "Basic Design Aspects of the 800 kV UHVDC Project Yunnan-Guangdong" Sixth International Conference of Power Transmission and Distribution Technology, Guangzhou 2007.
HVDC com Eliminação Seletiva de Harmônicos. 144 p. Dissertação (Mestrado) - Curso de Engenharia Elétrica, Universidade Federal de Minas Gerais, Minas Gerais, 2012.

Boylestad, R. L. Introdução á analise de circuitos. $8^{\circ}$ Edição, [S.1.]: Prentice Hall do Brasil LTDA, 1997.

Carvalho, A. P. S. D. de. Analise Técnico-Econômica de linhas de transmissão em corrente continua em alta tensão : estudos de casos. Dissertação (Monografia) — Universidade Federal do Rio de Janeiro, Rio de Janeiro, Agosto 2012.

Conceição, R. G. Transmissão de Energia Elétrica em Ultra Alta Tensão: O Complexo Hidreletrico Belo Monte. 2015. 69f. TCC (Graduação) - Curso de Engenharia Elétrica, Centro Tecnológico, Universidade Severino Sombra, Vassouras, 2015.

EPE. Balanço Energético Nacional 2011. Empresa de Pesquisa Energética. Brasília, 2011.

EPE. Balanço Energético Nacional 2015. Empresa de Pesquisa Energética. Brasília, 2015.

EPE. Projeto da usina hidrelétrica de Belo Monte. Empresa de Pesquisa Energética. Brasília, 2011.

Gussow, M. Eletricidade Básica - 2ª edição, Pearson, 1997.

Haeusler, M. Second International Symposium on Standards for ultra high voltage transmission. In: Equipment Design for the Yunnan-Guangdong UHVDC Project. Germany: [s.n.], 2008.

Haeusler, M.; Huang, H.; Papp, K. Design and testing of $800 \mathrm{kv}$ hvdc equipment. In: Cigre Conference. PARIS: [s.n.], 2008.

Kimbark, E. W. Direct Current Transmission. [S.1.]: Wiley interscience, 1971.

Machado, A. M. F; Pereira, B. R; Ramos, L. D. O. Usina Hidrelétrica Belo Monte: Implicações Institucionais, Econômicas e Sociombientais. 2011. 21 p. Produção de caso - Escola de direito de São Paulo - Fundação Getulio Vargas, São Paulo, 2011.

Nunes, R. R. Coordenação De Isolamento Para Transitórios De Manobra Considerando A Forma De Onda Das Sobretensões. 2006. 122 f. Dissertação (Mestrado) - Curso de Pós Graduação em Engenharia Elétrica, Universidade Federal de Minas Gerais, Belo Horizonte, 2006.

Reis, R. S. Transmissão De Energia Elétrica Em Corrente Contínua: Estudo Geral E Análise De Casos. 2013. 44f. TCC (Graduação) - Curso de Engenharia Elétrica, Centro Tecnológico, Universidade Severino Sombra, Vassouras, 2013

Rima. Linha de transmissão 800kv kingu-estreito. Relatório de impacto ambiental. Altamira, 2015.

Sato, A. K. C. Transmissão de Potência em Corrente Contínua e Corrente Alternada: Estudo Comparativo. 2013. 90 f. TCC (Graduação) - Curso de Engenharia Elétrica, Universidade Estadual Paulista, Guaratinguetá, 2013.

Silva, M. T. F. D.. Projeto \& Desenvolvimento de um padrão para Alta Tensão em Corrente Contínua na Faixa de $1 \mathrm{kV}$ a 50 kV. 2011. $84 \mathrm{f}$. Dissertação (Mestrado) - Curso de Pós- Graduação em Metrologia Pontifica Universidade Católica do Rio de Janeiro, Rio de Janeiro, 2011.

www.aneel.gov.br, Agencia Nacional de energia elétrica, acesso em $25 / 10 / 2015$. 